\title{
Comment travailler sur les attentats terroristes en classe de langue?
}

Gérald Schlemminger

\section{(2) OpenEdition \\ 12 Journals}

Édition électronique

URL : http://journals.openedition.org/esp/1549

DOI : 10.4000/esp.1549

ISSN : 2532-0319

Éditeur

Centre d'Information sur l'Éducation Bilingue et Plurilingue

Édition imprimée

Date de publication : 1 décembre 2017

Pagination : 53-63

ISSN : 1127-266X

\section{Référence électronique}

Gérald Schlemminger, "Comment travailler sur les attentats terroristes en classe de langue? »,

Éducation et sociétés plurilingues [En ligne], 43 | 2017, mis en ligne le 01 février 2019, consulté le 30 avril 2019. URL : http://journals.openedition.org/esp/1549; DOI : 10.4000/esp.1549 


\section{COMMENT TRAVAILLER SUR LES ATTENTATS TERRORISTES EN CLASSE DE LANGUE?}

\section{Gérald ScHLEMMINGER}

In quanto formatore di francese lingua straniera in un istituto di insegnamento universitario in Germania accompagno una volta alla settimana $i$ nostri studenti, futuri insegnanti di francese lingua straniera in Germania in una scuola per un esercizio d'applicazione. A turno ogni studente fa lezione; l'insegnante di ruolo ed io li osserviamo. Dopo la lezione ci riuniamo per un dare un riscontro sul modo in cui la lezione si è svolta. L'assenza di una studentessa ci ha portati a sostituirla su due piedi. Questa "lezione di sostituzione" si è svolta una settimana dopo un fatto sconvolgente, gli attentati terroristici a Parigi, il 13 novembre 2015. Questi avvenimenti sono stati presi come argomento della lezione.

Parole chiave: didattica del francese lingua straniera, civiltà, terrorismo

As teacher-trainer of French as a Foreign Language in a German institution of higher education, once a week we accompany our students, future FFL teachers in Germany, to the school were they practice their pedagogy. Each student in turn teaches the class while the official teacher and I look on and take notes. After the class, we talk it over with the student-teacher. When, one day, the designated student-teacher was absent, we took her place on the spur of the moment. This «substitute class» took place the week following the terrible news event: the terrorist attacks in Paris on November 13, 2015. This was the subject we dealt with in this class.

Keyzords: didactics of French as a Foreign Language, civilization, terrorism

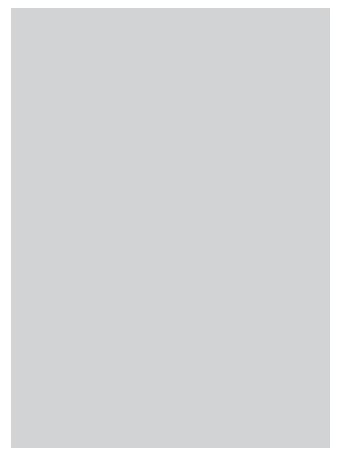

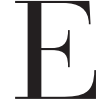

n tant que formateur de FLE (français langue étrangère) dans un établissement d'enseignement universitaire en Allemagne, la Pädagogische Hochschule de Karlsruhe, nous accompagnons une fois par semaine nos étudiants, futurs enseignants de FLE en Allemagne, dans une école d'application. À tour de rôle, l'un-e des ćtudiant-c-s fait cours; l'enscignant titulaire de la classe de français et nous-même l'observons. Après le cours, nous nous réunissons pour faire un retour sur la manière dont la séance s'est déroulée.

L'absence d'une étudiante nous a amené à la remplacer au pied levé devant les élèves. Ce «cours de remplacement» a eu lieu une semaine après une actualité bouleversante, les attentats terroristes à Paris, le 13 novembre 2015. Ces événements ont été repris comme sujet du cours. 
Comment travailler sur les attentats terroristes en classe de langue?

G. SChlemmingeR

QUAND L'ACTUALITÉ ENTRE EN GLASSE DE FRANÇAIS LANGUE ÉTRANGÈRE

\section{LE «DÉGLENCHEUR} DE LA PAROLE»
Avant de présenter et analyser cette séance - qui, pour des raisons de formation, a été filmée - nous préciserons quelques invariants pédagogiques qui guident notre formation.

Nous présenterons les différentes phases du cours de FLE comprenant l'expression du ressenti des élèves en classe de $5^{\mathrm{e}}$ (《7. Klasse») en rapport avec les attentats du 13 novembre 2015 à Paris, la co-écriture d'un texte résumant ce vécu et la lecture-traduction d'un témoignage d'une victime des attentats.

La classe est composée de 19 élèves (10 garçons, 9 filles), âgés de 12 à 13 ans. Onze d'entre eux ont l'allemand comme première langue, 8 le turc. Ils suivent les cours de français depuis un an et trois mois et sont de niveau Al (utilisateur élémentaire) selon le Cadre européen commun de référence pour les langues. Quinze élèves sont d'origine turque, issus de la $2^{\mathrm{e}}$ ou $3^{\mathrm{e}}$ génération d'immigration, ils sont de culture musulmane. Une élève est voilée. Les élèves sont motivés, participent, en général, activement au cours; bien intégrés, ils maitrisent les codes et normes du système scolaire allemand. Ce sont des parents de la classe moyenne de Karlsruhe, ville de taille moyenne, qui envoient leurs enfants dans ce collège-lycée turc privé, reconnu par l'Etat fédéral de Bade-Wurtemberg.

Notre rôle habituel, dans cette classe, est celui de l'observateur qui accompagne ses étudiants, au nombre de cinq, lors de leur stage en pratique accompagnée. Nous sommes six jours après les attentats du 13 novembre 2015 à Paris (pour l'accueil des élèves après les attentats, voir aussi Dubois \& Sambe 2016).

Ce sont les circonstances exceptionnelles - maladie d'une étudiante et évènement sociétal exceptionnel - qui me poussent à vouloir assurer ce cours. Dans cette situation dramatique que vit mon pays, il me semble impensable de voir faire une leçon du manuel. Cela dit, j'ai vu la 7. Klasse seulement 5 fois comme observateur, je ne connais pas le nom de la plupart des élèves; je n'ai pas pu mettre en place des lieux d'échange, des «institutions» de travail coopératif, etc. - Je me lance... J'ai juste le temps de choisir, d'une part, ce qu'on appelle en didactique de langue un «déclencheur de parole» - ici une image symbolisant les attentats à Paris (voir fig. 1) - et, d'autre part, la réaction à chaud d'une victime des attentats.

Afin de pouvoir mieux communiquer, nous demandons aux élèves de disposer leurs chaises en cercle. Puis nous distribuons une petite bande de scotch et quelques stylos feutres pour que chaque élève y écrive son prénom et la colle sur sa poitrine.

Afin de pouvoir échanger et communiquer avec les élèves, il me semble indispensable que je puisse les appeler par leur prénom. Je me colle également une bande de scotch 
Comment travailler sur les attentats terroristes en classe de langue?

G. SCHLEMMINGER
L'ACCUEIL DES ÉMOTIONS ET DES RESSENTIS

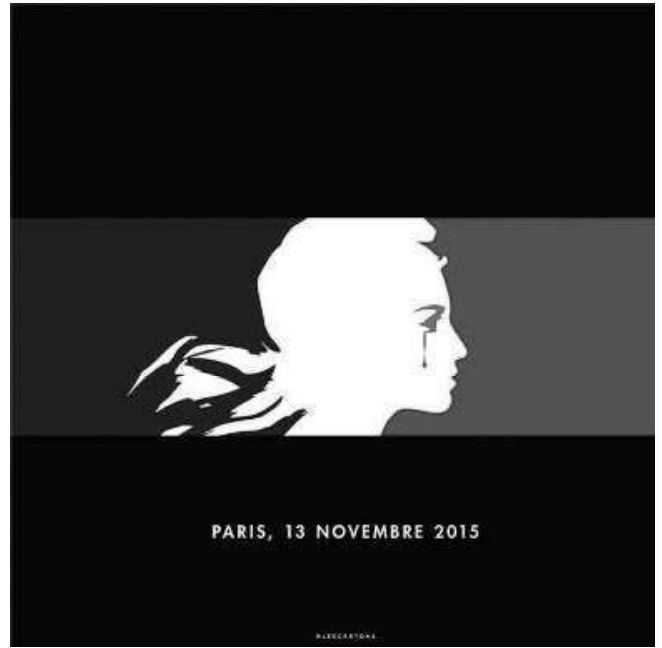

Figure 1 : Marianne qui pleure (Paris, éditions LESCARTONS)

sur ma veste.

Cette mise en place dure quelques minutes. Nous posons ensuite le transparent de l'affiche «Marianne qui pleure» (fig. 1) sur le rétroprojecteur. Les élèves réagissent aussitôt et disent en français ou en allemand qu'il s'agit des attentats à Paris. Ils interprètent correctement le sens sémiotique de l'affiche: les couleurs de la France, le sous-titre qui fait référence à la date de l'attentant; une personne qui pleure, le noir comme couleur du deuil. Qui est la personne qui pleure? Nous expliquons que c'est une femme, Marianne, symbole de la France, du peuple français.

Comme je ne suis pas sûr d'être compris, je dis que le symbole de l'Allemagne est l'aigle et demande quel est le symbole de la Turquie. Les élèves répondent: le croissant et l'étoile.

De façon un peu pesante et professorale, nous introduisons le sujet du cours: «Aujourd'hui, on va travailler sur l'attaque terroriste à Paris». Pendant que nous préparons le transparent pour noter les réactions des élèves, nous entendons certains dire:

- «Das haben die absichllich gemachbs (Ils [les terroristes] l'ont fait exprès)

- «Das haben die gemacht, damit die Franzosen mehr Angst haben» (Ils l'ont fait pour que les Français aient [encore] plus peur).

Nous enchaînons en reprenant et amplifiant le propos: «Bonne idée: "Ils l'ont fait exprès". "Das haben sie absichtlich gemacht.»

Comme je souhaite davantage connaître leur pensée, je pose des questions et l'échange suivant 
Comment travailler sur les attentats terroristes en classe de langue?

G. SCHLemmingeR s'établit:

- «Que pensez-vous de cette attaque? Êtes-vous tristes?»

Nous notons et reprenons en français ce que disent les élèves:

- «ife suis triste, weil so viele gestorben sind.» (... parce que beaucoup [de personnes] sont mortes)

- «fe suis triste parce que die böse sind.» (... parce qu'ils sont méchants)

-Nous: «Qui est méchant, Arcan?» (les noms des élèves ont été changés)

- «Les terroristes»

Par ma question "Êtes-vous tristes?" j’induis de la compassion. J'aurais pu demander ce qu'ils ressentent face à l'attaque. Je ne le fais pas compte tenu de leur niveau de langue élémentaire et sachant qu'ils ont déjà travaillé sur l'expression d'émotions comme rige suis tristes.

Ensuite, Aslan dit (en allemand) que lorsqu'il y a des morts à Paris, tout le monde en parle. Mais dans d'autres pays, beaucoup plus de gens meurent (par des actes de guerre ou de terrorisme) et personne ne s'y intéresse.

J'accueille ce désarroi, le note tout en lui demandant à quels pays il pense.

- «Syrie wegen Krieg, Irak, Mali, Palestine» (La Syrie, à cause de la guerre...).

Je sens qu'Aslan formule un sentiment d'injustice. Il me semble important non seulement d'accueillir ces propos mais, dans la mesure du possible, d'y apporter des éléments de compréhension:

- Nous: «La question se pose: pourquoi parle-t-on de Paris? Pourquoi on ne parle pas du Mali? Pourquoi on ne parle pas de la Palestine? Pourquoi on parle de Paris.»

Des réponses fusent:

- «Wir wissen es halt nicht.» (Mais on ne le sait pas...).

- «Doch.» (Sil).

- «Paris ist in Europa. Also haben die Menschen... [inaudible]» (Paris est en Europe.

Alors les gens...).

- Aslan: «Oh! Aber Amerika... [inaudible]» (Mais les États-Unis...).

- Beria: «Aber das ist ein Grund. Paris hat es auch für Amerika gemacht, als mal was in Amerika war. Und weil die denken, dass wir daran... [inaudible]» (Mais ce n'est pas une raison. Paris a [aidé] les États-Unis quand il y avait des évènements... Et parce qu'ils pensent que c'est notre...).

Il m'est difficile d'ordonner ce flot de réactions, d'autant plus que je suis obligé de choisir celles qui me sont facilement et rapidement transposables dans un français simple et compréhensible pour les élèves. Fe sélectionne du débat lidée qui se profile pour moi comme un argument pertinent par rapport à l'intervention d'Aslan, celui du (mort/kilomètres: "plus les victimes semblent éloignées, moins elles susciteront d'empathies. Je transpose cette notion abstraite dans un contexte concret et facilement accessible et note sur le transparent:

- «On parle de Paris parce que Paris est à $600 \mathrm{~km}$ de Karlsruhe». Et nous ajoutons oralement: 
Comment travailler sur les attentats terroristes en classe de langue?

G. SCHLemmingeR
- «La Syrie, c'est à $3000 \mathrm{~km}$, la Palestine est à $4000 \mathrm{~km}$. L'Irak est à $4000 \mathrm{~km}$. C'est une explication. Comme c'est très loin, on en parle moins; on en parle plus lorsque les évènements sont proches».

- Ensuite, Beria apporte l'information qu'en Allemagne, des terroristes ont été arrêtés au départ d'un avion. Elle continue:

- "Es ist schon wichtig, darïber zu berichten, damit jeder weiss, was passiert." (Il est très important d'en informer [les gens] afin que tout le monde sache ce qui se passe.)

Elle ajoute par là un argument supplémentaire concernant l'attitude de la presse et des médias face aux attaques.

Je note sur le transparent seulement le fait qu'elle relate et non son argument, car je n'arrive pas à trouver une transposition simple en français.

Aslan intervient de nouveau:

- (Il y a quelques jours, les?? ont fait?? parce qu'ils pensaient que ce sont de mauvais Musulmans.)

Nous accueillons ses propos:

- «On va le noter: "Des personnes pensent que les Musulmans sont des terroristes"». Et nous ajoutons les commentaires: «C'est faux! C'est absurde».

Aslan exprime bien le désarroi des élèves face à l'amalgame très répandu entre la religion musulmane et les attaques. Par la suite, Selay apporte une explication pour faire la différence entre «croyants» et «terroristes». Nous la notons en français:

- «Les croyants (musulmans, chrétiens, juifs...) ne tuent pas. Les terroristes ne sont pas des croyants. Ils sont manipulés».

Je suis gêné par cette simplification d'un phénomène sociétal très complexe. Mais je ne sais pas comment le rendre compréhensible autrement dans un français facile. Pour éviter de le ramener au seul islam, j'apporte une généralisation en incluant toutes les religions monothéistes.

Cet échange a duré une vingtaine minutes. Nous apportons le titre «Paris pleure» au texte.

Je choisis ce titre pour faire le lien entre l'affiche «Marianne qui pleures et le ressenti ainsi que les interrogations des éleves. À la fin de cette longue phase d'echanges et d'écriture collective, ma concentration baisse (et celle des élèves aussi).

Le résumé de l'échange avec les élèves donne la co-construction du texte suivant: Aslan intervient de nouveau: - «Vor ein paar tagen haben [inaudible] gemacht, weil sie dachten, dass die Mosleme schlecht sind.» - (Il y a quelques jours, les?? ont fait?? parce qu'ils pensaient que ce sont de mauvais Musulmans.)

Nous accueillons ses propos:

- «On va le noter: "Des personnes pensent que les Musulmans sont des terroristes"». Et nous ajoutons les commentaires: «C'est faux! C'est absurde». 
Comment travailler sur les attentats terroristes en classe de langue?

G. SChlemmingeR

(FIG. 2)

«PARIS PLEURE» TEXE COMMUN

DE LA GLASSE 7

\section{L'APPROPRIATION DU TEXTE GOLLECTIF}

Aslan exprime bien le désarroi des élèves face à l'amalgame très répandu entre la religion musulmane et les attaques. Par la suite, Selay apporte une explication pour faire la différence entre «croyants» et «terroristes». Nous la notons en français:

- «Les croyants (musulmans, chrétiens, juifs...) ne tuent pas. Les terroristes ne sont pas des croyants. Ils sont manipulés».

Je suis gêné par cette simplification d'un phénomène sociétal très complexe. Mais je ne sais pas comment le rendre compréhensible autrement dans un français facile. Pour éviter de le ramener au seul islam, j'apporte une généralisation en incluant les autres religions monothéistes.

Cet échange a duré une vingtaine minutes. Nous apportons le titre «Paris pleure» au texte.

Je choisis ce titre pour faire le lien entre l'affiche «Marianne qui pleures et le ressenti ainsi que les interrogations des élèves. À la fin de cette longue phase d'échanges et d'écriture collective, ma concentration baisse (et celle des élèves aussi).

Le résumé de l'échange avec les élèves donne la co-construction du texte suivant:

«L'attentat des terroristes se passe le vendredi 13 novembre 2015.

Je suis triste parce que des Français sont morts (les mots nouveaux sont soulignés). Je suis triste parce que les terroristes sont méchants. Ils ont tué 130 personnes.

Il y a aussi beaucoup d'autres morts dans le monde. Mais on ne parle pas de ces morts en Syrie, en Turquie, au Mali, en Palestine, en Irak, en Ukraine... On parle de Paris parce que Paris est à $600 \mathrm{~km}$ de Karlsruhe.

En Allemagne, la police a aussi arrêté des terroristes.

Des personnes pensent que les Musulmans sont des terroristes. C'est faux! C'est absurde. Les croyants (musulmans, chrétiens, juifs...) ne tuent pas. Les terroristes ne sont pas des croyants. Ils sont manipulés».

Précisons que ce long échange, résumé en français dans un texte coconstruit, ne correspond pas au dialogue maieutique cher à la didactique des langues, technique qui consiste à amener l'apprenant à exprimer un (pré-)savoir qui permet de construire par la suite un nouveau savoir (langagier plus particulièrement).

D'un point de vue pédagogique, il nous semble important de s'approprier ensemble ce texte pour saisir sa cohérence et éventuellement l'amender. La première phase consiste à relire ensemble, phrase par phrase, le texte. Comme il s'agit d'apprenants débutants, nous donnons le modèle, en insistant plus particulièrement sur la prosodie de l'énoncé, entre autres, pour apporter l'emphase, voire la gestuclle où 
Comment travailler sur les attentats terroristes en classe de langue?

G. SChlemmingeR

UN TÉMOIGNAGE DE FACEBOOK cela est nécessaire, par exemple: «C'est faux!» / «Les terroristes ne sont pas des croyants».

À la lecture du texte, je me sens, de nouveau, gêné par la simplification d'une pensée complexe: certes, les élèves musulmans de la classe se disent tous croyants. Cela dit, on peut appartenir à une culture (chrétienne, musulmane...), tout en étant laïc et respecter les règles fondamentales de la vie en société. Ici, je ne me sens pas capable d'aborder cette question d'une façon simple. En fait, la classe abordera ce sujet plus loin, au hasard de la prononciation du mot «musulmans...

Les élèves répètent la phrase ou l'énoncent individuellement ou collectivement si la prononciation d'un mot s'avère difficile. C'est le cas de la voyelle nasale du mot «musulman». Nous introduisons un petit exercice: «Es-tu musulman?» Réponse attendue: «Oui, je suis musulman. / Non, je ne suis pas musulman». Rolf, d'origine allemande, répond: «Non, je suis athéiste». À l'instar de Rolf, nous avons l'impression que les élèves confondent les notions de «culture» et de «religion».

Je décide alors d'intervenir en allemand pour préciser les choses:

- «On peut ne pas croire en Dieu tout en appartenant à une culture. Tu es, certes, athée, mais ta culture est chrétienne. Tu es de culture chrétienne, mais tu ne crois pas en Dieu. Tout comme on peut être musulman et athée».

La lecture continue. La diphtongue /o-y/ du mot «croyant» pose également un problème de prononciation. Nous reprenons le même type d'exercice que précédemment: «Es-tu croyant?» Réponse attendue: «Oui, je suis croyant(e). / Non, je ne suis pas croyant[e].Je suis athée»).

Je suis frappé par le fait que les notions smusulmans et scroyants ne posent pas uniquement un défi au niweau phonétique, ce sont également les représentations qui posent le plus de questions aux élèves.

À la fin de la lecture, nous décidons que c'est le moment de faire une pause de dix minutes. Nous demandons ensuite aux élèves de recopier le texte dans leur cahier. Nous notons les mots nouveaux au tableau:

croyant: je suis croyant

tuer: ils ont tué 130 personnes

mort: il y a beaucoup de morts

Nous leur demandons de les copier dans le cahier de vocabulaire dont dispose chaque élève. Pendant ce temps, nous distribuons l'affiche «Marianne qui pleure» aux élèves. Certains ayant recopié le texte plus vite que d'autres peuvent alors la coller sous le texte.

Afin d'apporter de nouvelles perspective quant à l'attentat, nous avons voulu présenter un témoignage d'une victime (indirecte), Antoine Leiris, journaliste sur France Info, qui a perdu son épouse, Hélène, tuée au Bataclan (voir le texte en annexe).

C'est par les réseaux sociaux que j'ai pris connaissance de ce texte. Fe l'ai choisi parce que sa charge émotionnelle m'a touché. 
Comment travailler sur les attentats terroristes en classe de langue?

G. SCHLemmingeR

\section{VOUS N'AUREZ PAS} MA HAINE

\section{LIMITES ET}

POSSIBILITÉS D'UNE ÉCOUTE ACTIVE EN CLASSE DE LANGUE
Le texte étant trop difficile pour des apprenants d'un niveau A1, nous l'avons simplifié tout en essayant d'en garder l'aspect très personnel et émotionnel (voir figure 3). Il nous était important de garder le titre. Comme il est d'une complexité langagière que les élèves ne maîtrisent pas, nous avons mis en sous-titre deux possibles traductions allemandes:

«Vous n'aurez pas ma haine» (Ich werde Euch nicht hassen / Ihr werdet nicht meinen Hass haben.)

«Vendredi soir, vous avez volé la vie de ma femme, l'amour de ma vie, la mère de mon fils. Mais vous n'aurez pas ma haine.

Non, je ne vous fais pas ce cadeau: vous n'aurez pas ma haine. Vous voulez que j’ai peur. Vous voulez que j'ai peur de mes amis. Vous avez perdu. Je reste libre.

Ma femme est morte et je suis très, très triste. Elle est très belle. Je sais qu'elle est avec moi, avec mon fils Melvil, avec nous. Je sais que nous nous verrons au paradis.

Nous sommes deux, mon fils et moi. Nous sommes plus forts que toutes les armées du monde.

Maintenant, je vais voir Melvil, mon fils. Il dort. Il a 17 mois. Il va manger, comme tous les jours. Et nous allons jouer, comme tous les jours. Ce petit garçon est heureux et libre. Non, vous n’aurez pas sa haine».

Figure 3 Page Facebook, réduction didactique du texte «Vous n'aurez pas ma haine» d'A. Leiris (2015)

Nous avions initialement l'intention de faire un travail de compréhension de l'écrit de façon traditionnelle: lecture du texte - élucidation des mots inconnus par le biais d'un dialogue maïeutique avec les élèves - compréhension globale et détaillée. Mais nous avons sous-estimé le temps nécessaire à l'écriture et l'appropriation de notre texte collectif. Sur les 90 minutes, il nous reste à peine une dizaine de minutes. Nous optons alors pour la traduction.

Kasim s'étonne - roon Facebook?» - que nous utilisions en classe un texte authentique et qui vient tout juste d'être publié. Nous expliquons qu'Antoine Leiris existe réellement et que c'est lui qui a écrit le texte:

- «Tu peux chercher son nom sur Facebook et tu trouveras le texte. Pour le travail en classe, pour que vous le compreniez mieux, j’ai simplifié le texte».

Nous sommes surpris de la bonne compréhension et de la bonne traduction des propos d'Antoine Lciris. Güzid propose une traduction du titre qui nous paraît mieux rendre l'intention de l'auteur que ce que nous avions proposé: - «Ihr verdient nicht meinen Hass.»

J'ai vécu ce cours comme un moment exceptionnel de vie de la classe, dû à un évènement exceptionnel.

D'un point de vue didactique, il est également hors cadre à plusieurs titres:

- Le séquencement en phases: la didactique du FLE (en Allemagne) 
Comment travailler sur les attentats terroristes en classe de langue?

G. SCHLemmingeR propose de changer d'activité toutes les 5 à 7 minutes (pour garder l'attention des élèves). Ce cours de 90 minutes compte 5 phases là où on en aurait pu en attendre une bonne quinzaine.

- L'expression orale des élèves en langue française: les élèves ont participé très activement au cours, souvent en langue allemande.

- Certes, les instructions du Bade-Wurtemberg prévoient pour le français la «médiation», c'est-à-dire le résumé en langue de scolarisation d'un contenu en langue étrangère. Mais, ici, nous avons travaillé sur une traduction littérale et détaillée du texte de Facebook (1).

Il nous semble que cette façon de procéder, la démarche, l'approche devrait être la règle à l'école, en classe de langue: par une écoute active, l'enseignant part des centres d'intérêt des élèves, de leur vécu, de leurs expériences, de leur esprit curieux. Un manuel, une méthode, quels qu'ils soient, ne peuvent répondre à cette approche (2) comme nous l'avons montré plus haut et comme C. Freinet l'avait déjà analysé en 1928 dans son écrit Plus de manuel. Il y fustige avec son emphase habituelle la relation duale traditionnelle entre élève et enseignant où ce dernier peine à instaurer un désir d'apprendre. À travers les techniques dites Freinet (l'imprimerie, la correspondance, le plan de travail, etc.) il prône la mise en place d'une relation médiatisée par un tiers, complétée par un accueil actif du désir de l'enfant. Le rôle de l'enseignant change dans cette triangulation, prend une autre direction.

Traditionnellement, l'enseignant, avec le soutien du manuel, apporte le savoir, détermine les objectifs, choisit les tâches, organise les apprentissages et sanctionne les résultats par des évaluations (sommatives). Que les guides de l'enseignant appellent désormais cette démarche «approche actionnelle» ne change en rien les pratiques d'enseignement et d'apprentissage comme nous l'observons régulièrement dans nos visites de classe: l'élève reste dans son rôle d'apprenant pris dans cette relation duale conflictuelle et suivant les étapes d'apprentissage fixées par l'enseignant.

Rappelons que rien ne s'oppose, dans le cadre scolaire actuel, à la mise en place d'un autre type de relation élève(s)-enseignant. Ce ne sont pas les schémas didactiques (3) et les objectifs linguistiques (lexicaux, grammaticaux...) ou communicationnels (actes de paroles, les quatre compétences...) qui doivent déterminer comment travailler sur un contenu, mais ce sont les contenus et les besoins de les communiquer à d'autres qui doivent conduire aux choix des techniques pédagogiques et à la manière d'organiser les apprentissages. Le rôle de l'enseignant supposé tout savoir peut évoluer vers celle/celui qui sait écouter et aider les élèves lorsqu'lis expriment leur désir (et également leur refus...) d'apprendre. Il mettra en place les outils, les «institutions» ( $f f$. A. Vasquez, F. Oury 1967) de médiation qui font évoluer cette relation duale vers l'émergence et le respect du désir d'apprendre des élèves. Elles sont au centre de l'acte éducatif, de l'intervention pédagogique. 
Comment travailler sur les attentats terroristes en classe de langue?

G. SCHLemmingeR

\section{BIBLIOGRAPHIE}

\section{ANNEXE}

Concluons avec une réflexion sur la nécessité d'une éducation à la citoyenneté. Afin qu'elle puisse s'accomplir, elle doit assurer, sur un plan symbolique, une fonction de coupure qui fasse repère, qui différencie les êtres et les choses ( $c$. R. Laffitte et le groupe VPI, 2006: 250).

DUBOIS A., SAMBE M. 2016. Accueillir les élèves après les attentats, Rerue Projet n 352 (juin): 28-32.

EDLER K. 2015. Islamismus als pädagogische Herausforderung. Stuttgart, Kohlhammer.

FREINET C. 1928. Plus de manuels scolaires. Méthode vivante et rationnelle de travail scolaire par l'Imprimerie à l'École. St. Paul, Éditions de l'Imprimerie à l'École. Dossier pédagogique de l'École Moderne n ${ }^{\circ} 7$.

LEIRIS A. 2015. «Vous n'aurez pas ma haine». Facebook. En ligne sur la page de l'auteur:

https://www.facebook.com/antoine.leiris/posts/1015445784999994 7?fref=nf\&pnref=story (consulté le: 12/12/2015).

LAFFITTE R. et le groupe VPI. 2006. Essais de pédagogie institutionnelle. L'école, un lieu de recours possible pour l'enfant et ses parents. Nîmes, Champ social.

OKAPI. 2015. Pourquoi y a-t-il des fanatiques. OKAPI (magazine pour adolescents), Paris (mars).

VASQUEZ A., OURY F. 1967. Vers une pédagogie institutionnelle. Paris, Maspero.

«Vendredi soir, vous avez volé la vie d'un être d'exception, l'amour de ma vie, la mère de mon fils mais vous n'aurez pas ma haine. Je ne sais pas qui vous êtes et je ne veux pas le savoir, vous êtes des âmes mortes. Si ce Dieu pour lequel vous tuez aveuglément nous a fait à son image, chaque balle dans le corps de ma femme aura été une blessure dans son cœur.

Alors non, je ne vous ferai pas ce cadeau de vous haïr. Vous l'avez bien cherché pourtant mais répondre à la haine par la colère, ce serait céder à la même ignorance qui a fait de vous ce que vous êtes. Vous voulez que j'aie peur, que je regarde mes concitoyens avec un œil méfiant, que je sacrifie ma liberté pour la sécurité. Perdu. Même joueur joue encore.

Je l'ai vue ce matin. Enfin, après des nuits et des jours d'attente. Elle était aussi belle que lorsqu'elle est partie ce vendredi soir, aussi belle que lorsque j'en suis tombé éperdument amoureux il y a plus de 12 ans. Bien sûr je suis dévasté par le chagrin, je vous concède cette petite victoire, mais elle sera de courte durée. Je sais qu'elle nous accompagnera chaque jour et que nous nous retrouverons dans ce paradis des 
Comment travailler sur les attentats terroristes en classe de langue?

G. SCHLemmingeR âmes libres auquel vous n'aurez jamais accès.

Nous sommes deux, mon fils et moi, mais nous sommes plus forts que toutes les armées du monde. Je n'ai d'ailleurs pas plus de temps à vous consacrer, je dois rejoindre Melvil qui se réveille de sa sieste. Il a 17 mois à peine, il va manger son goûter comme tous les jours, puis nous allons jouer comme tous les jours et toute sa vie ce petit garçon vous fera l'affront d'être heureux et libre. Car non, vous n'aurez pas sa haine non plus».

(A. Leiris, 2015)

\section{NOTES}

(1) Nous pourrons envisager des prolongements de ce cours. Comme les élèves viennent d'apprendre l'expression de son opinion («je pense que» / «je crois que» / «à mon avis»...), le présent travail pourrait être suivi d'un débat en français. - Voir aussi les propositions d'écoute active que propose K. Edler (2015). Le magazine pour adolescents OKAPI (mars 2015: 21-12) propose le texte «Pourquoi y a-t-il des fanatiques?». - Nous exclurions toutefois un travail grammatical sur les deux textes du cours. Nous ne suivons pas la méthodologie didactique qui voudrait que tout «document de base» serve comme outil pour entrainer le plus de compétences possibles. Le texte devient alors un simple prétexte pour justifier des activités pédagogiques.

(2) Nuançons notre propos: La nouvelle méthode FLE en Suisse alémanique Mille feuilles (Großenbacher 2012) n'a plus de documents de base et est orientée, dans l'organisation pédagogique de ses activités, vers une tâche finale qui est toujours une production à exposer (devant le groupe-classe, à l'extérieur de la classe...); environ cinq activités différentes, parmi lesquelles l'élève ou un groupe d'élèves peuvent choisir une ou deux, préparent à la production finale. Il s'agit d'une véritable approche actionnelle soutenue par les techniques de la pédagogie différenciée et des outils issus de l'éducation nouvelle, comme un plan de travail. Mais de tels manuels et méthodes, révolutionnaires au sens propre, font exception dans le paysage de la didactique des langues. Elles bouleversent trop l'agencement classique des phases, le rôle et la fonction de l'enseignant, le sens des apprentissages.

(3) Dans les méthodes allemandes en FLE, il s'agit des phases suivantes: introduction des mots nouveaux - première écoute du document sonore - question de l'enseignant pour vérifier la compréhension globale du contenu - deuxième écoute du document sonore - ... Elles se trouvent, à quelques variantes près, dans toutes les méthodes de FLE pour un niveau A1 / A2. 